\title{
Sphenochoanal polyp: a rare entity with review of literature
}

\begin{abstract}
Sphenochoanal polyp is a rare form of choanal polyp which originates from the sphenoid sinus and extends to posterior choanae via sphenoethmoidal recess. Its occurrence is extremely rare. They can be easily mistaken for antrochoanal polyp. Symptomatically, it is similar to antrochoanal polyp. So, adequate preoperative evaluation with diagnostic nasal endoscopy and computed tomography or magnetic resonance imaging is necessary for correct diagnosis and planning of appropriate surgical procedures. Surgical resection is the treatment modality of choice. We here present a case of sphenochoanal polyp along with review of literature.
\end{abstract}

Keywords: sphenochoanal polyp, endoscopic sinus surgery, sphenoid sinus
Volume 8 Issue 4 - 2017

\author{
Amber Kesarwani, Darwin Kaushal, Rahul \\ Kumar Singh, Amit Goyal \\ All India Institute of Medical Sciences, India
}

Correspondence: Amber Kesarwani, \#303, Resident Hostel, All India Institute of Medical Sciences, Jodhpur, Rajasthan, India , Tel+919057I04 I24, Email dramber.kesar@gmail.com

Received: June 08, 2017| Published: August 29, 2017

\section{Introduction}

Sphenochoanal polyps arise from the sphenoid sinus and pass through choanae to nasopharynx. It can be differentiated from antrochoanal polyp by endoscopic examination, CT scan and/or MRI. ${ }^{1}$ Sphenochoanal polyps are of uncertain etiology. ${ }^{2}$ Due to its relatively deep seated location, it results in a late presentation. Proper clinical and radiologic evaluation should be done in such cases before planning surgical treatment. The rarity of the isolated sphenoid sinus lesion and important anatomical relation of sphenoid sinus lesions necessitates investigation for underlying pathology, especially malignancy and the origin and extent of the mass prior to the surgical excision. ${ }^{3}$

\section{Case report}

A 27year old female presented in OPD with complaint of right side nasal obstruction since two years with mouth breathing and snoring since 1year. Symptoms were gradually progressive. There were no allergic symptoms like rhinorrhea or recurrent sneezing. Her anterior rhinoscopy examination was normal, but on diagnostic endoscopic examination, pale whitish polyp was seen, which on decongestion was seen coming through right sphenoethmoid recess and nasopharynx. Left choanae was also blocked by the mass. The right and left middle meatus were clear of any pathological changes (Figure 1). Paranasal sinuses Computed tomography showed hypodense soft tissue density mass lesion with CT attenuation of 20-30 HU filling right sphenoidal sinus and extending through sphenoidal ostium, across sphenoethmoidal recess into nasopharynx causing obstruction of both posterior choana and extending upto posterior pharyngeal wall (Figure 2). The polyp was removed via endoscopic transnasal approach. The polyp was found to attach to the junction of lateral wall and floor of sphenoid. The attachement was identified and the polyp was removed en block from its stalk and the stalk was debrided. Histopathological examination of the specimen revealed as inflammatory polyp (Figure $3)$.

\section{Discussion}

Nasal polyps are benign nasal mass and usually arise from paranasal sinuses and extends towards choana and then to nasopharynx. ${ }^{4}$ Its nomenclature is made according to the sinus that it is originated from. Choanal polyps account $4-6 \%$ of the all nasal polyps. ${ }^{4}$ Most commonly it is derived from the maxillary sinus antrum i.e. Antrochoanal polyp.
Ethmochoanal and Sphenochoanal are rare types of polyp arising from ethmoids and sphenoid respectively.

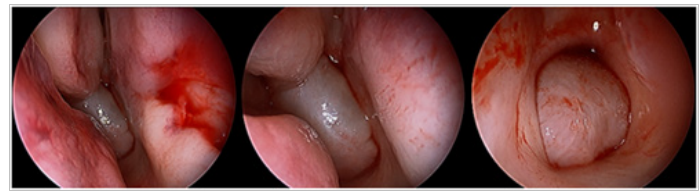

Figure I From left to right, sphenochoanal polyp arising through right sphenoethmoidal recess and obstructing both choanae. Right middle meatus can be seen free from disease.

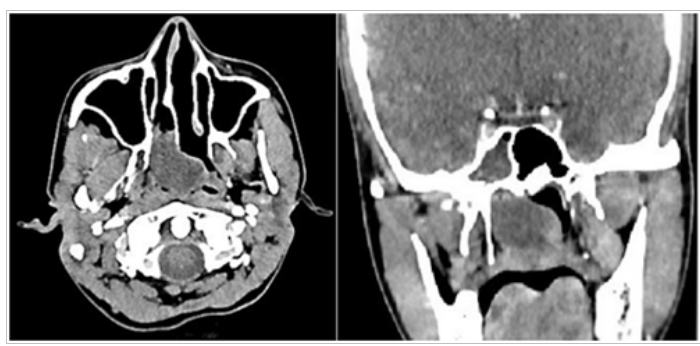

Figure 2 CT Paranasal sinus - axial and coronal section reveals soft tissue density filling right sphenoid sinus and extending to nasopharynx obstructing bilateral choana.

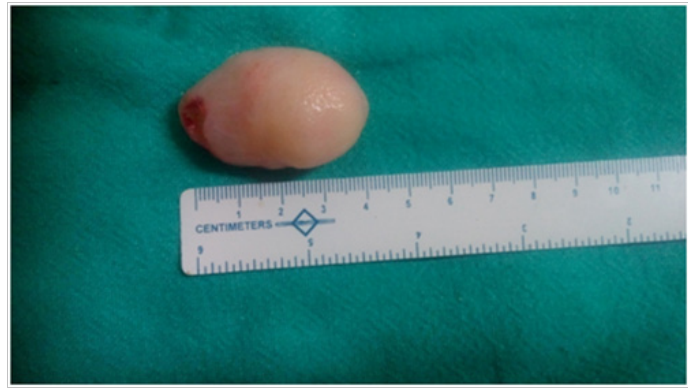

Figure 3 Specimen of spheno choanal polyp after excision.

Sphenochoanal polyps have been reported for the first time by Zuckerkandl. ${ }^{5}$ It is a rare disorder. ${ }^{2}$ More than $50 \%$ of SCPs are observed in children. ${ }^{6}$ It consists of solitary mass that arises from sphenoid sinus and then towards the choana through its drainage ostium, going through sphenoethmoidal recess occasionally reaching nasopharynx pushing inferior soft palate. ${ }^{1,2}$ 
Histologically, choanal polyps are similar. They are formed by a cystic center surrounded by oedema stroma which presents a kind of infiltration of inflammatory cells. Its surface is covered by respiratory epithelium ${ }^{1}$ where areas of metaplasia can be found. ${ }^{8}$ In our patient histopathological specimen revealed inflammatory polyp.

Sphenochoanal polyp should be distinguished from meningoencephalocele (presents as skull base defect that allows communication between cerebral and nasal cavity), nasopharyngeal angiofibroma presents with recurrent epistaxis), inverted papillomas (presents as unilateral tumor with enlargement of osteomeatal complex in older patients) among other nasal masses. ${ }^{1}$ However, its main differential diagnosis is antrochoanal polyps as they are clinical and histologically indistinctive.

Attempts have been made to link it with IgE- mediated allergies. However, allergic patients are not predisposed to choanal polyp formation. Most patients are totally free of their rhinologic symptoms after surgical removal of choanal polyp. ${ }^{9}$ Laboratory investigation including RAST testing for specific allergens and skin prick tests has yielded the general impression that choanal polyp is unrelated to allergy. ${ }^{8}$ However, in a study association between allergy and formation of choanal polyp is established. ${ }^{10}$ The etiology of SCP is not clearly defined, but chronic obstruction of the sinus ostia, as well intramural cysts originating from sinuses are blamed as causative factors. ${ }^{7}$

The clinical symptoms of Sphenochoanal polyps are almost invariable in respect to antrochoanal polyps, but there are differences in terms of the endoscopic examination and paranasal CT findings. Although the major symptom of patients with sphenochoanal polyps is nasal obstruction that can be unilateral or bilateral; runny nose, headache can also occur and sometimes patients present to the physician with complaint of snoring. In our case, the chief complaint of the patient was nasal obstruction. It was unilateral initially but gradually obstruction became bilateral. However, she also had complaints of mouth breathing and snoring. Due to its deep seated location, there is tendency of these polyps to be missed on anterior rhinoscopy. In our case, polyp was not appreciated on anterior rhinoscopic examination. The patient also had Right nasal spur and was misdiagnosed as right deviated nasal septum as the cause for nasal obstruction. Thus, in these patients, nasal endoscopic is very essential and mandatory, not only to confirm the diagnosis but also to visualise the extent of the polyp. However, CT scan is the gold standard examination. Thus, History, physical examination and radiologic findings lead us to the diagnosis. The combined use of imaging techniques and diagnostic nasal endoscopy is suggested for accurate diagnosis of isolated sphenoid sinus lesions so that the patient can be correctly diagnosed and also get benefitted by minimally invasive techniques.
Transnasal endoscopic resection can be a quite safe method of treatment in such cases. The incidence of recurrence can be prevented by complete removal of polyp which includes intranasal as well as intrasphenoidal component also. Regular follow-up is essential.

\section{Conclusion}

Choanal polyps are the most common diagnosis to be considered in cases of progressive unilateral nasal obstruction. Although antrochoanal polyp is most common type of choanal polyp, sphenochoanal polyp is one entity which is always to be keep among differentials. The combination of history, nasal endoscopic examination and radiology leads us to diagnosis. Transnasal endoscopic resection is treatment of choice.

\section{Conflicts of interest}

Author declares there are no conflicts of interest.

\section{Funding}

None.

\section{References}

1. Tosun F, Yetiser S, Akcam T, et al. Sphenochoanal polyp: endoscopic surgery. Int J Pediatr Otorhinolaryngol. 2001;58(1):87-90.

2. Dadas B, Yilmaz O, Vural C, et al. Choanal polyp of sphenoidal origin. Eur Arch Otorhinolaryngol. 2000;257(7):379-381.

3. Nayak DR, Pujary K, Valiathan M, et al. Sphenochoanal polyp with heterotopic glial tissue. J Laryngol Otol. 2007;121(3):274-276.

4. Ozbay M, Yorgancilar E, Kinis V, et al. A rare cause of nasal obstruction: Sphenochoanal polyp. J Clin Exp Invest. 2012;3:435-437.

5. Mills CP. Secretory cysts of the maxillary antrum and their relation to the development of antrochoanalpolypi. J Laryngol Otol. 1959;73(5):324-334.

6. Ileri F, Köybaşioĝlu A, Uslu S. Clinical presentation of a sphenochoanal polyp. Eur Arch Otorhinolaryngol. 1998;255(3):138-139.

7. Berg O, Carenfelt C, Silfverswärd C, et al. Origin of the choanal polyp. Arch Otolaryngol Head Neck Surg. 1988;114(11):1270-1271.

8. Crampette L, Mondain M, Rombaux P. Sphenochoanal polyp in children. Diagnosis and Treatment. Rhinology. 1995;33(1):43-45.

9. Soh KBK, Tan KK. Sphenochoanal polyps in Singapore: Diagnosis and current management. Singapore Med J. 2000;41(4):184-187.

10. Cook PR, Davis WB, McDonald R, et al. Antrochoanal polyps: a review of 33 cases. Ear Nose Throat J . 1993;72(9):632. 